\title{
On gauge fields in fluid turbulence
}

\author{
T. H. Moulden \\ The University of Tennessee Space Institute, USA
}

\begin{abstract}
A standard gauge velocity field is introduced into the constant density NavierStokes equations and extended to treat fluid turbulence. Some of the elementary properties of such gauge fields are explored and relationships between them established. In particular, the gauge fields separate out the irrotational motion from the vortical motion. An example that describes the inactive component of a turbulent flow is presented.
\end{abstract}

Keywords: gauge fields, turbulence models, energy estimates.

\section{Background}

Write the field equations for the flow of a constant density viscous fluid in the standard form:

$$
\frac{\partial v_{i}}{\partial x_{i}}=0 ; \quad \frac{\partial v_{i}}{\partial t}+\frac{\partial}{\partial x_{j}}\left(v_{i} v_{j}\right)+\frac{\partial P}{\partial x_{i}}=\nu \frac{\partial^{2} v_{i}}{\partial x_{j} \partial x_{j}}+f_{i}
$$

where $P(\mathbf{x}, t)$ is the pressure field normalized by the constant fluid density. Equations $(1 a, b)$ define the field $(\mathbf{v}, P)$ in which $\mathbf{v}(\mathbf{x}, t)$ is the instantaneous velocity field at location $\mathbf{x}$ and time $t$. $\mathbf{f}(\mathbf{x})$ denotes a time independent body force in equation $(1 b)$. Herein, the kinematic viscosity coefficient, $\nu$, is taken to be constant. To be useful these equations must possess a unique regular solution for meaningful boundary conditions; and the existence of such a solution is assumed in the following discussion. Certain additional assumptions must be made before equations $(1 a, b)$ can be adopted to describe the turbulent flow of a viscous fluid. These assumptions include the need for the turbulent velocity and pressure fluctuations to be continuous and have bounded energy. It is also required that all velocity-related correlation functions be bounded. As is standard, the divergence of equation $(1 b)$ produces a Poisson equation to compute the pressure field directly:

$$
\frac{\partial^{2} P}{\partial x_{i} \partial x_{i}}=\frac{\partial f_{i}}{\partial x_{i}}-\frac{\partial^{2}}{\partial x_{i} \partial x_{j}}\left(v_{i} v_{j}\right)
$$


since, as stated above, it is assumed that all required derivatives exist. Some regularity results for the Navier-Stokes equations are given in Seregin [1].

Let the Reynolds decomposition be expressed in the standard form:

$$
\mathbf{v} \mapsto \mathbf{V}+\mathbf{u} ; \quad \mathbf{V}=\mathcal{E}(\mathbf{v}) \text { and } \mathcal{E}(\mathbf{u})=0
$$

if $\mathbf{u}(\mathbf{x}, t)$ is the fluctuating velocity field and $\mathbf{V}(\mathbf{x}, t)$ the corresponding mean velocity. Equation (2) implies that the kinetic tensor, $\mathbf{R}=\mathbf{v} \otimes \mathbf{v}$ will be decomposed as $\mathbf{R} \mapsto \overline{\mathbf{R}}+\mathcal{R}$ in the mean motion equations provided that $\overline{\mathbf{R}}=\mathbf{V} \otimes \mathbf{V}$ denotes the mean kinetic tensor. $\mathcal{R}(\cdot)$ in the above represents an appropriate mean value operator whose specific form is not of significance for the present discussion and $\mathcal{R}=\mathcal{E}(\mathbf{u} \otimes \mathbf{u})$ is referred to as the Reynolds tensor. As is well known, introduction of the Reynolds decomposition renders equations $(1 a, b)$ unclosed and hence unsolvable without an additional statement in the form of a turbulence model: $\chi(\mathcal{R}, \mathbf{V}, \boldsymbol{\alpha})=\mathbf{0}$ (with a vector, $\boldsymbol{\alpha}$, of scalar constants). The constraints that must be placed upon such models, are not the present interest for some of which see the discussion in Moulden [2]. Details of specific turbulence models are given in such references as Chen and Jaw [3], Wilcox [4] as well as in Launder and Sandham [5]. See also the discussion in Gatski [6].

Start by noting some fundamental properties of equations $(1 a, b)$ that will be required for subsequent developments:

a) As is standard, equations $(1 a, b)$, being based upon Newtonian mechanics, are covariant under the Galilean group, $\mathbb{G}_{a}$ :

$$
\mathbf{x}^{*}=\mathbf{Q}\left[\mathbf{x}+\mathbf{V}_{T} t+\mathbf{x}_{0}\right] ; \quad t^{*}=t+t_{0}
$$

which implies a velocity transformation of the form $\mathbf{v}^{*}=\mathbf{Q}\left[\mathbf{v}+\mathbf{V}_{T}\right]$. Here, $\mathbf{Q} \in \mathbb{S O}_{3}$ is a constant coordinate rotation while $\mathbf{V}_{T}$ is the constant Galilean boost velocity while $\mathbf{x}_{0}$ and $t_{0}$ are constant space and time translations. The pressure field is invariant across inertial frames. Finally, note that the spatial and temporal gradients transform as:

$$
\frac{\partial(\cdot)}{\partial x_{i}^{*}} \equiv Q_{i j} \frac{\partial(\cdot)}{\partial x_{j}} ; \quad \frac{\partial(\cdot)}{\partial t^{*}}=\frac{\partial(\cdot)}{\partial t}-\left(V_{T}\right)_{k} \frac{\partial(\cdot)}{\partial x_{k}}
$$

under the group $\mathbb{G}_{a}$. The Reynolds averaged equations, like the Navier-Stokes equations, are fully covariant under the Galilean group.

b) Gurtin [7] gave a simple uniqueness result for the constant density NavierStokes equations $(1 a, b)$ under the assumption that certain regularity conditions are satisfied. In particular, that the norm of the stretching tensor $\mathbf{D}=\operatorname{sym}(\mathbf{L})$ (if $\mathbf{L}=\boldsymbol{\nabla}(\mathbf{v})$ is the velocity gradient tensor) must be bounded for all $\mathbf{x}, t$ of interest. This constraint implies that $\mathbf{D}$ must have bounded eigenvalues: a condition related to the requirements on the boundedness of the Reynolds tensor $\mathcal{R}$. The theorem of Gurtin [7] showed that the velocity field, $\mathbf{v}(\mathbf{x}, t)$, must be unique but that the pressure field has the uniqueness property only up to an arbitrary function of time:

$$
P(\mathbf{x}, t) \mapsto P(\mathbf{x}, t)+P^{*}(t)
$$

say. This finding is nothing more than noting that the simple equality:

$$
\frac{\partial}{\partial x} P(\mathbf{x}, t)=\frac{\partial}{\partial x}\left[P(\mathbf{x}, t)+P^{*}(t)\right]
$$


must hold for any bounded function $P^{*}=P^{*}(t)$, only. Most important for practical applications is the velocity field uniqueness obtained from the theorem of Gurtin [7] and this result will be required herein.

It will be found below that certain gauge transformations introduced in the literature do not possess full Galilean covariance and so are not, to this extent, consistent with the Navier-Stokes equations. Some applications of one such gauge transformation will, however, be explored.

\section{On the Reynolds decomposition}

The instantaneous Navier-Stokes equations for constant density flow were written in equations $(1 a, b)$ (and formally constitute a well posed problem when appropriate boundary and initial conditions are appended: even though certain issues relating to uniqueness and regularity remain unresolved). The instantaneous kinetic tensor $\mathbf{R}=\mathbf{v} \otimes \mathbf{v}$, again normalized by the fluid density, has been introduced in equation $(1 b)$. Being symmetric the kinetic tensor has real eigenvalues one of which is $\lambda=\langle\mathbf{v}, \mathbf{v}\rangle$, the kinetic energy. It is assumed here that the velocity field is regular in that all required derivatives exist. The same is assumed of the pressure field.

With these definition in place the mean motion equations, corresponding to equations $(1 a, b)$, can be written as:

$$
\frac{\partial \mathbf{V}}{\partial t}+\operatorname{div}(\overline{\mathbf{R}})+\operatorname{div}(\mathcal{R})+\nabla(\bar{P})=\nu \nabla^{2}(\mathbf{V})+\mathbf{f} ; \quad \operatorname{div}(\mathbf{V})=0 \quad(3 a, b)
$$

when the velocity field $\mathbf{v}(\mathbf{x}, t)$ has been decomposed in the form $\mathbf{v} \mapsto \mathbf{V}+\mathbf{u}$ with $|\mathbf{u}|$ bounded. The corresponding equation for the fluctuating velocity, $\mathbf{u}(\mathbf{x}, t)$, with $\operatorname{div}(\mathbf{u}) \equiv 0$, is given as:

$$
\frac{\partial \mathbf{u}}{\partial t}+\operatorname{div}[(\mathbf{V} \otimes \mathbf{u}+\mathbf{u} \otimes \mathbf{V})+\mathbf{u} \otimes \mathbf{u}-\mathcal{R}]+\nabla\left(p^{\prime}\right)=\nu \nabla^{2}(\mathbf{u})
$$

since the body force has been restrained as $\mathbf{f}=\mathbf{f}(\mathbf{x})$ only. Here the pressure field is given the usual decomposition as $P=\bar{P}+p^{\prime}$. The standard evolution equation for the Reynolds tensor $\mathcal{R}=\left(\mathcal{R}_{i j}\right)$ can be established from equation (4) and has the form:

$$
\frac{\partial \mathcal{R}_{i j}}{\partial t}+V_{k} \frac{\partial \mathcal{R}_{i j}}{\partial x_{k}}+\mathcal{R}_{j k} \frac{\partial V_{i}}{\partial x_{k}}+\mathcal{R}_{i k} \frac{\partial V_{j}}{\partial x_{k}}-\nu \frac{\partial^{2} \mathcal{R}_{i j}}{\partial x_{k}^{2}}=-\Psi_{i j}
$$

(see, for example, Frost and Moulden [8]). The definition:

$$
\Psi_{i j}=2 \nu \mathcal{E}\left[\frac{\partial u_{i}}{\partial x_{k}} \frac{\partial u_{j}}{\partial x_{k}}\right]+\frac{\partial}{\partial x_{k}} \mathcal{E}\left(u_{i} u_{j} u_{k}\right)+\left.\psi_{i j} \equiv \mathbf{E}_{T}\right|_{i j}+\left.\mathbf{D}_{F}\right|_{i j}+\psi_{i j}
$$

has been included in equation (5). In this equation $\mathbf{E}_{T}$ denotes the turbulence dissipation and $\mathbf{D}_{F}$ the velocity diffusion term whose explicit form is not of importance in the present context. As usual, turbulence models are required for both of these terms. Finally $\psi_{i j}$ denotes the standard pressure fluctuation terms in 
the Reynolds stress equation:

$$
\psi_{i j}=\mathcal{E}\left[\frac{\partial\left(u_{j} p^{\prime}\right)}{\partial x_{i}}+\frac{\partial\left(u_{i} p^{\prime}\right)}{\partial x_{j}}\right]-\mathcal{E}\left[p^{\prime}\left(\frac{\partial u_{j}}{\partial x_{i}}+\frac{\partial u_{i}}{\partial x_{j}}\right)\right]
$$

The velocity, $\mathbf{v}(\mathbf{x}, t)$, is unique and, for a given mean value operator, $\mathcal{E}$, so is the mean motion $\mathbf{V}$. Hence the fluctuations $\mathbf{u}(\mathbf{x}, t)$, must also be unique along with the Reynolds tensor $\mathcal{R}=\mathcal{E}(\mathbf{u} \otimes \mathbf{u})$ again when determined from the unique instantaneous velocity field defined by the Navier-Stokes equations. Under the group $\mathbb{G}_{a}$ the well known transformations:

$$
\mathbf{V}^{*}=\mathbf{Q}\left[\mathbf{V}+\mathbf{V}_{T}\right] ; \quad \mathbf{u}^{*}=\mathbf{Q} \mathbf{u}
$$

hold (see Speziale [11] for a more general discussion of the invariance properties of turbulence models). Only the mean velocity is influenced by the Galilean boost velocity $\mathbf{V}_{T}$. In other words, the turbulent fluctuations are transparent to uniform rectilinear motion of the inertial frame.

\section{A gauge decomposition}

The experimental work of Bradshaw [10] studied the inactive component of the motion external to a turbulent shear layer. This motion contains irrotational velocity fluctuations as well as pressure fluctuations and the two relate via a Bernoulli-type equation. It is appropriate to introduce a gauge field $\boldsymbol{\omega}(\mathbf{x}, t)$ that describes the irrotational motion. That is equation $(1 a)$, wherein $\operatorname{div}(\mathbf{v})=0$, allows the specification of a gauge velocity field $\boldsymbol{\omega}$ in the form: $\mathbf{v} \mapsto \mathbf{s}+\boldsymbol{\omega}$ when:

$$
\operatorname{div}(\mathbf{v})=0 \equiv \operatorname{div}(\mathbf{s})+\operatorname{div}(\boldsymbol{\omega})
$$

Since $\mathbf{v}$ is a function of space and time, it is assumed that $\mathbf{s}$ and $\boldsymbol{\omega}$ have that same dependence. The nature of the gauge field $\boldsymbol{\omega}(\mathbf{x}, t)$ is arbitrary within that constraint and could be stochastic. At this point there is nothing to specify that the above decomposition of the velocity field is unique. However, the variables $\mathbf{s}$ and $\boldsymbol{\omega}$ can at most, from equation $(1 d)$, differ by a divergence-free vector field. Clearly, when $\boldsymbol{\omega}=\boldsymbol{\nabla}(\varphi)$, for some scalar $\varphi$, the equality $\boldsymbol{\zeta}=\operatorname{curl}(\mathbf{v}) \equiv \operatorname{curl}(\mathbf{s})$ holds so that the $\mathbf{s}(\mathbf{x}, t)$ field captures all the vorticity in the flow. Now the condition $\operatorname{div}(\mathbf{v})=0$ provides the Poisson equation $\operatorname{div}(\mathbf{s})+\nabla^{2}(\varphi)=0$ and the $\boldsymbol{\omega}$ field is potential but not, in general, harmonic. Since the vector field $\operatorname{curl}(\mathbf{s})=\zeta$ is known a (nonunique) velocity field can be generated from it. There is non-uniqueness since any $\beta \in \mathbb{R}$ such that $\operatorname{curl}(\operatorname{grad}(\beta)) \equiv 0$ can be introduced additively. However, since $\operatorname{div}(\mathbf{v})=0$ has the physical meaning of mass conservation in constant density flow, the specification of $\mathbf{s}(\mathbf{x}, t)$ as the vorticity carrying component in the decomposition $\mathbf{v}=\mathbf{s}+\boldsymbol{\nabla}(\varphi)$ is unique: the scalar $\beta$ can be combined with $\varphi$. For a discussion of the more specific Stokes-Helmholtz decomposition, which is not required herein, see Wu et al. [12]. Throughout it is assumed that all vector fields are bounded and, in unbounded regions, tend to zero in the far field.

For the application in mind in section 5, it is appropriate to take the vector $\boldsymbol{\omega}$ to be potential since it can then represent the irrotational component of a turbulent flow; both inside and outside of a turbulent shear layer. The appropriate boundary conditions to be appended to the definition of the $\mathbf{s}$ and $\boldsymbol{\omega}$ fields will be addressed in section 5 for a specific flow field. 
From equation (1c) a Poisson equation for the pressure can be constructed, in the usual way, to give:

$$
\frac{\partial^{2} P}{\partial x_{i} \partial x_{i}}=\frac{\partial f_{i}}{\partial x_{i}}-\frac{\partial}{\partial x_{i}}\left(s_{j}+\omega_{j}\right) \frac{\partial}{\partial x_{j}}\left(s_{i}+\omega_{i}\right)
$$

once the $\mathbf{s}(\mathbf{x}, t)$ and $\boldsymbol{\omega}(\mathbf{x}, t)$ variables are known.

The above gauge decomposition was introduced by Weinan E and Liu [13] and discussed by Wang and Liu [14] in the context of numerical solutions of the Navier-Stokes equations. The latter adopted a Crank-Nicolson, or backward Euler, time discretization to construct a stable finite difference formulation. The objective herein is to explore the possible use of such gauge fields in turbulent flow: specifically to isolate the inactive motion from the vortical motion inside a turbulent shear layer. As noted above, these irrotational velocity fluctuations exist outside the shear layer and the $\omega$ field must also describe this external motion. Various aspects of the physics of the inactive motion are discussed in Bradshaw [10,15] and Phillips [16] when restricted to the case of a statistically stationary flow field. The present interest lies in exploring the extension to nonstationary turbulent flows. In particular, evolution equations for the inactive motion are required but now expressed in terms of the gauge fields.

From equations $(1 a, b)$ there is an evolution of the velocity field $\mathbf{s}(\mathbf{x}, t)$ and a specification of the gauge $\boldsymbol{\omega}(\mathbf{x}, t)$ given by the equations:

$$
\frac{\partial s_{i}}{\partial x_{i}}=-\frac{\partial \omega_{i}}{\partial x_{i}} \equiv-\frac{\partial^{2} \varphi}{\partial x_{i} \partial x_{i}} ; \quad \frac{\partial s_{i}}{\partial t}+\frac{\partial}{\partial x_{k}}\left[v_{i} v_{k}\right]=\nu \frac{\partial^{2} s_{i}}{\partial x_{k} \partial x_{k}}+f_{i}
$$

if the variable $\omega_{i} \equiv \partial \varphi / \partial x_{i}$ is potential, so that $\mathbf{v}=\mathbf{s}+\boldsymbol{\nabla}(\varphi)$, then the pressure field $P(\mathbf{x}, t)$ is determined retrospectively from the equality:

$$
\frac{\partial \varphi}{\partial t}+P=\nu \frac{\partial^{2} \varphi}{\partial x_{k} \partial x_{k}}
$$

once the gauge field $\varphi(\mathbf{x}, t)$ has been determined. The regularity assumptions made in the theory demand that both quantities, $\partial \varphi / \partial t$ and $\nabla^{2}(\varphi)$, are bounded. The pressure field is then well defined in equation (7). It hardly requires stating that splitting the velocity $\mathbf{v}$ into a rotational and irrotational parts is artificial in the sense that flow experiments do not allow this separation of the measured velocity field. If equation (7) is looked upon as a non-homogeneous equation for the potential $\varphi(\mathbf{x}, t)$ then the constraints to be imposed upon the function $P(\mathbf{x}, t)$, for a unique solution to exist, must be specified. From Stakgold [17] a boundedness condition on $P(\mathbf{x}, t)$ is required.

The potential $\varphi$ can be obtained from equation $(6 a)$ in terms of a Laplacian Green's function when the $\mathbf{s}(\mathbf{x}, t)$ field is known. If $\varphi(\mathbf{x}, t)$ is determined uniquely from equations $(6 a, b)$ then the pressure $P(\mathbf{x}, t)$ follows uniquely from equation (7). The classical vorticity transport equation for constant density fluid motion:

$\frac{\partial \zeta_{i}}{\partial t}+v_{j} \frac{\partial \zeta_{i}}{\partial x_{j}}=\frac{\partial v_{i}}{\partial x_{j}} \zeta_{j}+\nu \frac{\partial^{2} \zeta_{i}}{\partial x_{j} \partial x_{j}}+\epsilon_{i j k} \frac{\partial f_{k}}{\partial x_{j}} ; \quad \boldsymbol{\zeta}=\operatorname{curl}(\mathbf{v}) \equiv \operatorname{curl}(\mathbf{s})$

does not make reference to the fluid pressure. It is consistent with this observation that equation (7) determines the fluid pressure field from just the irrotational 
motion. Equation (V) is covariant under $\mathbb{G}_{a}$ since the transformation $\zeta^{*}=\mathbf{Q} \boldsymbol{\zeta}$ holds under that group.

Consider the Galilean transformation applied to the gauge decomposition introduced above when it is required that:

$$
\mathbf{s}^{*}=\mathbf{Q}\left[\mathbf{s}+\mathbf{V}_{T}\right] \quad \text { and } \quad \boldsymbol{\omega}^{*}=\mathbf{Q} \boldsymbol{\omega} \Rightarrow\left|\boldsymbol{\omega}^{*}\right| \equiv|\boldsymbol{\omega}|
$$

in addition $\left|\mathbf{s}^{*}\right|^{2} \leq\left[|\mathbf{s}|+\left|V_{T}\right|\right]^{2}$ as a bound on the vector $\mathbf{s}^{*}\left(\mathbf{x}^{*}, t^{*}\right)$ : a bound that must be boost velocity dependent. When $\boldsymbol{\omega}$ is potential there is the transformation: $\nabla^{*}\left(\varphi^{*}\right)=\mathbf{Q} \nabla(\varphi)$ under $\mathbb{G}_{a}$ so that $\left|\nabla^{*}\left(\varphi^{*}\right)\right|=|\nabla(\varphi)|$. It should be noted that the pair of equations $(6 \mathrm{~b}, 7)$ are not Galilean covariant under boosts since the transformed equation (6b) has the form:

$$
\frac{\partial s_{i}}{\partial t}+\frac{\partial}{\partial x_{j}}\left(v_{i} v_{j}\right)\left[+\left(V_{T}\right)_{j} \frac{\partial \omega_{i}}{\partial x_{j}}\right]=\nu \frac{\partial^{2} s_{i}}{\partial x_{j} \partial x_{j}}+f_{i}
$$

while equation (7) transforms to:

$$
\frac{\partial \varphi}{\partial t}\left[-\left(V_{T}\right)_{k} \frac{\partial \varphi}{\partial x_{k}}\right]+P=\nu \frac{\partial^{2} \varphi}{\partial x_{k} \partial x_{k}}
$$

to demonstrate the lack of Galilean covariance in the gauge transformation: the terms in brackets in equations $(\mathrm{Ga}, \mathrm{b})$ destroying the covariance. The equations $(6 b, 7)$ are, however, covariant under Galilean rotations. Equation (6a) is fully Galilean covariant. Equations (Ga,b) do not feature in the following discussion but must be recalled when transferring to a moving inertial frame.

\section{The gauge Reynolds decomposition}

Introduce a Reynolds decomposition into equations $(6 \mathrm{a}, \mathrm{b})$ by specifying that:

$$
\mathbf{S} \mapsto \mathbf{S}+\mathbf{r} ; \quad \varphi \mapsto \Psi+\phi
$$

where $\mathbf{S}=\mathcal{E}(\mathbf{s})$ and $\Psi=\mathcal{E}(\varphi)$. The vorticity field $\zeta(\mathbf{x}, t)=\operatorname{curl}(\mathbf{s})$ has two components: $\operatorname{curl}(\mathbf{S})$ and $\operatorname{curl}(\mathbf{r})$; the latter associating with the rotational part of the turbulent fluctuations. Given this decomposition, equation (6a) splits into:

$$
\frac{\partial S_{i}}{\partial x_{i}}=-\frac{\partial^{2} \Psi}{\partial x_{i} \partial x_{i}} ; \quad \frac{\partial r_{i}}{\partial x_{i}}=-\frac{\partial^{2} \phi}{\partial x_{i} \partial x_{i}}
$$

as statements of mean and fluctuating mass invariance in the current variables. Similarly equation (7) yields the pair of equations:

$$
\frac{\partial \Psi}{\partial t}+\bar{P}=\nu \frac{\partial^{2} \Psi}{\partial x_{i} \partial x_{i}} ; \quad \frac{\partial \phi}{\partial t}+p^{\prime}=\nu \frac{\partial^{2} \phi}{\partial x_{i} \partial x_{i}}
$$

for the mean and fluctuating pressure fields when the potentials $\phi$ and $\Psi$ are known. In addition, there is: $\mathcal{E}\left(p^{\prime} \mathbf{v}\right) \equiv \mathcal{E}\left(p^{\prime} \mathbf{r}\right)+\mathcal{E}\left(p^{\prime} \nabla(\phi)\right)$. Hence the component $\mathcal{E}\left(p^{\prime} \nabla(\phi)\right)$ depends only on the potential $\phi(\mathbf{x}, t)$. The component $\mathcal{E}\left(p^{\prime} \mathbf{r}\right)$, if it exists, is an interaction of the irrotational pressure fluctuations with the vortical 
flow field. Finally, equation (6b) implies the following:

$$
\left.\begin{array}{rl}
\frac{\partial S_{i}}{\partial t}+\frac{\partial}{\partial x_{k}}\left[\bar{R}_{i k}+\mathcal{R}_{i k}\right] & =\nu \frac{\partial^{2} S_{i}}{\partial x_{k} \partial x_{k}}+f_{i} \\
\frac{\partial r_{i}}{\partial t}+\frac{\partial}{\partial x_{k}}\left[V_{i} u_{k}+u_{i} V_{k}+u_{i} u_{k}-\mathcal{R}_{i k}\right] & =\nu \frac{\partial^{2} r_{i}}{\partial x_{k} \partial x_{k}}
\end{array}\right\}
$$

the field $\mathbf{r}(\mathbf{x}, t)$ being defined from equation (10b). Here $\mathcal{R}=\mathcal{E}(\mathbf{u} \otimes \mathbf{u})$, the original Reynolds tensor, relates directly to correlations of the $(\mathbf{r}, \phi)$ fluctuations through the above transformations. Explicitly there is:

$$
\mathcal{R}=\mathcal{P}+2 \mathcal{E}(\mathbf{r} \otimes \nabla(\phi))+\mathcal{E}(\boldsymbol{\nabla}(\phi) \otimes \nabla(\phi)), \quad \text { where } \mathcal{P}=\mathcal{E}(\mathbf{r} \otimes \mathbf{r})
$$

Equation ( $8 \mathrm{~b}$ ) now gives the potential $\phi(\mathbf{x}, t)$ uniquely from the Poisson Green's function in the form:

$$
\phi(\mathbf{x}, t)=-\int_{D(\xi)} G(\mathbf{x}, \boldsymbol{\xi})\left[\frac{\partial r_{i}(\boldsymbol{\xi}, t)}{\partial \xi_{i}}\right] d V(\xi)+\Gamma(\mathbf{x}, t)
$$

where $\Gamma(\mathbf{x}, t)$ represents the contribution from the boundary conditions. The corresponding time derivative can be obtained as:

$$
\frac{\partial \phi}{\partial t}=-\int_{D(\xi)} G(\mathbf{x}, \boldsymbol{\xi})\left[\frac{\partial^{2} r_{i}(\boldsymbol{\xi}, t)}{\partial t \partial \xi_{i}}\right] d V(\xi)+\frac{\partial \Gamma(\mathbf{x}, t)}{\partial t}
$$

The domain of integration only extends over the support of the field $\mathbf{r}(\mathbf{x}, t)$ and that function must be such that the integrals in equations $(11,11 a)$ are bounded. Given the value of $\partial \phi / \partial t$ from equation (11a) the fluctuating pressure is determined directly from equations $(8 b, 9 b)$. In both equations $(11)$ and $(11 a)$, the function $\mathbf{r}(\mathbf{x}, t)$ is only non-zero in any vortical region, such as turbulence, that is present in the domain of interest. The $\nabla(\phi)$ term follows from equation (11) as:

$$
\frac{\partial \phi(\mathbf{x}, t)}{\partial x_{i}}=-\int_{D(\xi)} \frac{\partial G(\mathbf{x}, \boldsymbol{\xi})}{\partial x_{i}}\left[\frac{\partial r_{j}(\boldsymbol{\xi}, t)}{\partial \xi_{j}}\right] d V(\xi)+\frac{\partial \Gamma(\mathbf{x}, t)}{\partial x_{i}}
$$

for each component of $\mathbf{x}$.

Similar statements hold for the mean motion potential $\Psi(\mathbf{x}, t)$ in equations $(8 a)$ and $(9 a)$. That is, for example:

$$
\Psi(\mathbf{x}, t)=-\int_{D(\xi)} G(\mathbf{x}, \boldsymbol{\xi})\left[\frac{\partial S_{i}(\boldsymbol{\xi}, t)}{\partial \xi_{i}}\right] d V(\xi)+\Lambda(\mathbf{x}, t)
$$

again with the integral taken over the support of the $\mathbf{S}(\mathbf{x}, t)$ function. Derivatives of $\Psi(\mathbf{x}, t)$ then follow as above.

The relationship between the different velocity decompositions that have been introduced above needs to be clarified in order to establish the gauge Reynolds decomposition equations. From the above definitions:

$$
\mathbf{v}=\mathbf{V}+\mathbf{u}=\mathbf{s}+\boldsymbol{\omega} \equiv \mathbf{S}+\mathbf{r}+\nabla(\Psi)+\nabla(\phi)
$$


It then follows from application of the operator $\mathcal{E}(\cdot)$ that:

$$
\mathbf{u}=\mathbf{r}+\boldsymbol{\nabla}(\phi) \quad \text { and } \quad \mathbf{V}=\mathbf{S}+\boldsymbol{\nabla}(\Psi)
$$

to define the fluctuating and mean velocity fields. Associated with these velocity fields are the fluctuating and mean vorticity fields:

$$
\boldsymbol{\zeta}^{\prime} \equiv \operatorname{curl}(\mathbf{u})=\operatorname{curl}(\mathbf{r}) \text { and } \bar{\zeta} \equiv \operatorname{curl}(\mathbf{V})=\operatorname{curl}(\mathbf{S})
$$

such that the $\mathbf{r}(\mathbf{x}, t)$ and $\mathbf{S}(\mathbf{x}, t)$ vector fields carry the vorticity of the turbulent fluctuations and the mean motion respectively. The irrotational motion is described by the mean, $\nabla(\Psi)$, and fluctuating, $\nabla(\phi)$, potential fields. The vortical motion, $\mathbf{r}(\mathbf{x}, t)$ and $\mathbf{S}(\mathbf{x}, t)$ act as sources for the inactive components $\phi(\mathbf{x}, t)$ and $\Psi(\mathbf{x}, t)$ in equations $8(a, b)$. The above Reynolds decomposition calls forth the transformations:

$$
\mathbf{S}^{*}=\mathbf{Q}\left[\mathbf{S}+\mathbf{V}_{T}\right] ; \quad \mathbf{r}^{*}=\mathbf{Q} \mathbf{r} ; \quad \boldsymbol{\nabla}^{*}\left(\Psi^{*}\right)=\mathbf{Q} \boldsymbol{\nabla}(\Psi) ; \quad \boldsymbol{\nabla}^{*}\left(\phi^{*}\right)=\mathbf{Q} \boldsymbol{\nabla}(\phi)
$$

under the Galilean group.

Equation (10b) allows the construction of an equation for the Reynolds gauge stress $\mathcal{P}_{i j}=\mathcal{E}\left(r_{i} r_{j}\right)$ associated with the vortical component of the flow. This requires the evaluation of correlations of the form:

$$
\mathcal{E}(\mathbf{u} \otimes \mathbf{r}) \equiv \mathcal{E}(\mathbf{r} \otimes \mathbf{r})+\mathcal{E}(\mathbf{r} \otimes \nabla(\phi))
$$

wherein the first term will, generally, predominate as discussed by Bradshaw [10]. Specifically, there is:

$$
\frac{\partial \mathcal{P}_{i j}}{\partial t}+V_{k} \frac{\partial \mathcal{P}_{i j}}{\partial x_{k}}+\Pi_{i j}=\nu \nabla^{2}\left(\mathcal{P}_{i j}\right)-2 \nu \mathcal{E}\left(\frac{\partial r_{i}}{\partial x_{k}} \frac{\partial r_{j}}{\partial x_{k}}\right)
$$

where $\Pi_{i j}=\Sigma_{\alpha=1,7} \Pi_{i j}^{(\alpha)}$, with the individual contributions given as:

$$
\begin{array}{rlr}
\Pi_{i j}^{(1)} & =\mathcal{P}_{j k} \frac{\partial V_{i}}{x_{k}}+\mathcal{P}_{i k} \frac{\partial V_{j}}{\partial x_{k}} ; \quad \Pi_{i j}^{(2)}=V_{k} \mathcal{E}\left[r_{j} \frac{\partial^{2} \phi}{\partial x_{i} \partial x_{k}}\right]+V_{k} \mathcal{E}\left[r_{i} \frac{\partial^{2} \phi}{\partial x_{j} \partial x_{k}}\right] \\
\Pi_{i j}^{(3)} & =\frac{\partial V_{i}}{\partial x_{k}} \mathcal{E}\left[r_{j} \frac{\partial \phi}{\partial x_{k}}\right]+\frac{\partial V_{j}}{\partial x_{k}} \mathcal{E}\left[r_{i} \frac{\partial \phi}{\partial x_{k}}\right] ; & \Pi_{i j}^{(4)}=\mathcal{E}\left[\frac{\partial\left(r_{i} r_{j} r_{k}\right)}{\partial x_{k}}\right] \\
\Pi_{i j}^{(5)} & =\mathcal{E}\left[r_{j} r_{k} \frac{\partial^{2} \phi}{\partial x_{i} \partial x_{k}}+r_{i} r_{k} \frac{\partial^{2} \phi}{\partial x_{j} \partial x_{k}}\right] ; & \Pi_{i j}^{(6)}=\mathcal{E}\left[\left[\frac{\partial\left(r_{i} r_{j}\right)}{\partial x_{k}}\right] \frac{\partial \phi}{\partial x_{k}}\right] \\
\Pi_{i j}^{(7)} & =\mathcal{E}\left[\left[r_{j} \frac{\partial^{2} \phi}{\partial x_{k} \partial x_{i}}+r_{i} \frac{\partial^{2} \phi}{\partial x_{k} \partial x_{j}}\right] \frac{\partial \phi}{\partial x_{k}}\right]
\end{array}
$$


If the correlations between the vortical and irrotational components are neglected then the quantity $\boldsymbol{\Pi}$ in equation (13) reduces to: $\Pi=\Pi^{(1)}+\Pi^{(4)}$ only. Equation (13) then takes on the form:

$\frac{d \mathcal{P}_{i j}}{d t}+\mathcal{P}_{j k} \frac{\partial V_{i}}{\partial x_{k}}+\mathcal{P}_{i k} \frac{\partial V_{j}}{\partial x_{k}}+\mathcal{E}\left[\frac{\partial\left(r_{i} r_{j} r_{k}\right)}{\partial x_{k}}\right]=\nu \nabla^{2} \mathcal{P}_{i j}-2 \nu \mathcal{E}\left[\frac{\partial r_{i}}{\partial x_{k}} \frac{\partial r_{j}}{\partial x_{k}}\right]$

which can be compared to equation (5). It is seen that equation (5) contains the pressure term $\psi_{i j}$ (given explicitly in equation (5a)) which is absent from (13a). This is due to the structure of the gauge decomposition revealed in equations $(9 b)$ and $(10 b)$. The pressure field does, in this gauge formulation, only relate directly to the irrotational part of the flow field. It can be noted that the linearized compressible flow equations (see Goldstein [18], for example) contain a similar splitting of the velocity field but with the possibility of vorticity production in higher order terms. However, the present discussion is restricted to constant density motion and, as such, there are no entropy changes to take into account (and no pressure component in the Beltrami diffusion equation to consider). The limit $\rho \rightarrow$ const of the compressible Navier-Stokes equations is not a trivial limit; see the discussion in Schochet [19] for the special case of a baratropic fluid. The relationship between the compressible and incompressible flow theories is not direct.

\section{An example: inactive motion}

It is well established that not all velocity fluctuations in a turbulent shear layer are vortical in nature. As mentioned above these irrotational velocity fluctuations, and the associated pressure fluctuations, propagate outside the turbulent shear layers. See the discussion in Bradshaw [10,15] and Phillips [16]. The present interest lies in adopting the gauge fields introduced above to discuss the physics of the inactive motion both inside and outside a turbulent shear layer. Moyal [20] showed that in wave number space the pressure associates with velocity components that reside in the span of the wave number vector. A significant noise field arises in high speed turbulent shear layers as discussed, for example, in Phillips [21] and Tam [22] (with the latter showing photographs of Mach wave propagation external to a supersonic jet flow).

Outside the shear layer the equality $\mathbf{r} \equiv \mathbf{0}$ holds, but irrotational fluctuations do exist in that region where the quantity $\nabla(\phi)$ is non-zero. Phillips [16] took the irrotational nature of the inactive motion (when statistically stationary) to imply that it could be explained in terms of a potential. Hence, under these assumptions, a solution of a Laplace equation was sought for that potential. The theory of Phillips [16] gave a $1 / x^{4}$ decay of the energy $\mathcal{E}\left(u_{i} u_{i}\right)$ into the far field that was confirmed by the experiments reported by Bradshaw [15]. However, such a formulation does not allow the local time dependent external flow field to be described. The equations developed above allow a more general description of the irrotational motion. 
Equations for region (A):

Refer to Figure 1 when the equations for region $(A)$ are given as:

$$
\left.\begin{array}{rlrl}
\frac{\partial S_{i}}{\partial t}+\frac{\partial}{\partial x_{k}}\left[\bar{R}_{i k}+\mathcal{R}_{i k}\right] & =\nu \frac{\partial^{2} S_{i}}{\partial x_{k} \partial x_{k}} \\
\frac{\partial r_{i}}{\partial t}+\frac{\partial}{\partial x_{k}}\left[V_{i} u_{k}+u_{i} V_{k}+u_{i} u_{k}-\mathcal{R}_{i k}\right] & =\nu \frac{\partial^{2} r_{i}}{\partial x_{k} \partial x_{k}} \\
\frac{\partial \Psi}{\partial t}+\bar{P} & =\nu \frac{\partial^{2} \Psi}{\partial x_{i} \partial x_{i}} ; & \frac{\partial \phi}{\partial t}+p^{\prime} & =\nu \frac{\partial^{2} \phi}{\partial x_{i} \partial x_{i}} \\
\frac{\partial r_{i}}{\partial x_{i}} & =-\frac{\partial^{2} \phi}{\partial x_{k} \partial x_{k}} ; & \frac{\partial S_{i}}{\partial x_{i}} & =-\frac{\partial^{2} \Psi}{\partial x_{k} \partial x_{k}}
\end{array}\right\}
$$

The body force $\mathbf{f}(\mathbf{x})$ has not been included in the present discussion. Equations $(11 a, 14 e)$ allow the pressure fluctuations in region $(\mathrm{A})$ to be given explicitly as:

$$
p^{\prime}(\mathbf{x}, t)=-\nu \operatorname{div}(\mathbf{r})+\int_{\mathcal{D}(\xi)} G(\mathbf{x}, \boldsymbol{\xi})\left[\frac{\partial^{2} r_{i}(\boldsymbol{\xi}, t)}{\partial t \partial \xi_{i}}\right] d V(\xi)-\frac{\partial \Gamma(\mathbf{x}, t)}{\partial t}
$$

when $\operatorname{div}(\mathbf{r})$ is computed from equation $(14 b)$. Now it is found that the local pressure fluctuations in region (A) depend upon the local value of $\operatorname{div}(\mathbf{r})$ and an integral over the domain defined by the support of $\mathbf{r}$. In addition, there is in equation (15) a local contribution from the boundary conditions. This contribution will vanish for steady flow. The mean pressure, both inside and outside the shear layer, follows in the same way from equation ( $9 a$ ) when the gauge function $\Psi(\mathbf{x}, t)$ is determined from equation ( $8 a)$ and the function $\mathbf{S}(\mathbf{x}, t)$ from equation (10a).

Equations for region (B):

The mean external flow is assumed to lack a vortical component so that the $(B)$ region flow is defined by the system:

$$
\left.\begin{array}{rlrl}
\mathbf{V} & =\nabla(\Psi) ; & \mathbf{u} & =\nabla(\phi) \\
\frac{\partial \Psi}{\partial t}+\bar{P} & =\nu \frac{\partial^{2} \Psi}{\partial x_{i} \partial x_{i}} ; & \frac{\partial \phi}{\partial t}+p^{\prime} & =\nu \frac{\partial^{2} \phi}{\partial x_{i} \partial x_{i}} \\
0 & =-\frac{\partial^{2} \phi}{\partial x_{k} \partial x_{k}} ; & \frac{\partial S_{i}}{\partial x_{i}} & =-\frac{\partial^{2} \Psi}{\partial x_{k} \partial x_{k}}
\end{array}\right\}
$$

Note that equations $(14 c, d)$ and $(16 c, d)$ are identical so that boundary conditions $\nabla(\phi)=0$ are specified at $x_{2}=0$ and in the limit $x_{2} \rightarrow \infty$ and a smooth solution can be sought on the whole domain. Equations (14d) and (16d) determine the fluctuating pressure field both inside, and external to, the turbulent shear layer once the potential $\phi(\mathbf{x}, t)$ has been determined. At the surface $x_{2}=0$ there is $\mathbf{v} \equiv \mathbf{0}$ which implies that both equalities $\mathbf{V}=\mathbf{0}$ and $\mathbf{u}=\mathbf{0}$ must hold. It further follows that $\mathbf{r}=\mathbf{0}$ while $\boldsymbol{\nabla}(\phi)=\mathbf{0}$ is also true on this surface. The condition $\mathbf{r}=\mathbf{0}$ also holds at the edge of the shear layer $x_{2}=L$. It is assumed that all required fields are specified at the upstream boundary $x_{1}=0$ (which is taken to be some convenient origin for the computation as shown on Figure 1). 


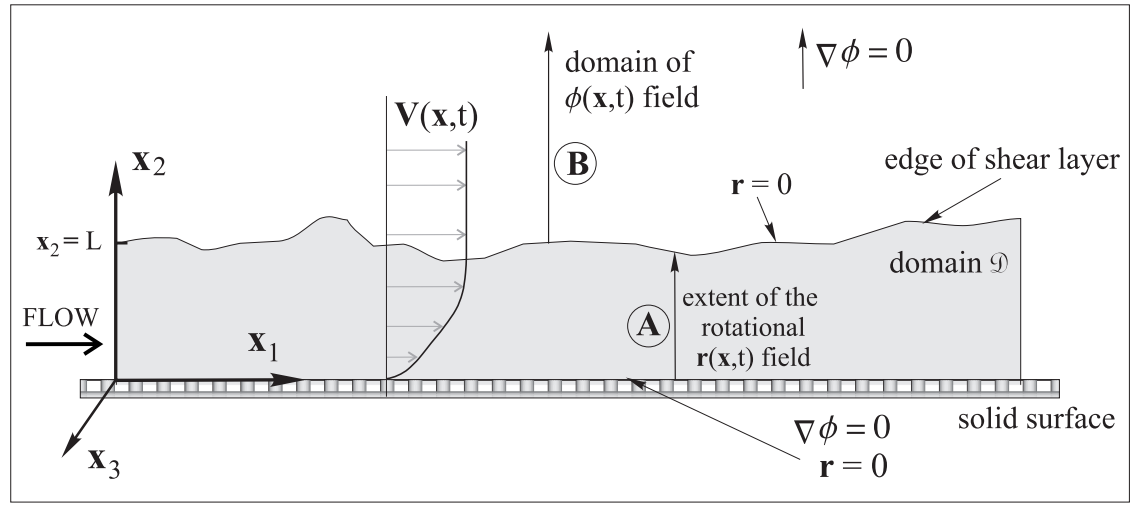

Figure 1: Regions in the flow field near a turbulent shear layer.

Equation (15) also defines the pressure fluctuations in the region external to the shear layer where, however, the local $\operatorname{div}(\mathbf{r})$ term is identically zero. Alternatively, equation $(16 d, 16 e)$ define $p^{\prime}=-\partial \phi / \partial t$ directly when equation (11a) applies. The integration is still over the support of the rotational motion but now the $(\mathbf{x}, t)$ values refer to this external region. For steady boundary conditions, the intensity of the pressure fluctuations external to the shear layer is given explicitly as:

$$
\mathcal{E}\left(p^{\prime} p^{\prime}\right)=\int_{\mathcal{D}} \int_{\mathcal{D}} G(\mathbf{x}, \boldsymbol{\xi}) G(\mathbf{x}, \boldsymbol{\eta}) \mathcal{E}\left[\frac{\partial^{2} r_{i}}{\partial t \partial \xi_{i}} \frac{\partial^{2} r_{j}}{\partial t \partial \eta_{j}}\right] d V(\eta) d V(\xi)
$$

and a similar expression for the velocity correlation can be obtained from equation $(11 b)$. That is, without the contribution for the boundary conditions the correlation:

$$
\mathcal{E}\left(\frac{\partial \phi}{\partial x_{i}} \frac{\partial \phi}{\partial x_{j}}\right)=\int_{\mathcal{D}} \int_{\mathcal{D}} \frac{\partial G(\mathbf{x}, \boldsymbol{\xi})}{\partial x_{i}} \frac{\partial G(\mathbf{x}, \boldsymbol{\eta})}{\partial x_{j}} \mathcal{E}\left[\frac{\partial r_{k}}{\partial \xi_{k}} \frac{\partial r_{m}}{\partial \eta_{m}}\right] d V(\eta) d V(\xi)
$$

is obtained which, from the form of the Green's function, varies like $1 / x_{2}^{4}$ for large distances from the shear layer. Pressure velocity correlations follow in the same way and need not be written down.

\section{Final remarks}

It has been shown that a non-Galilean invariant gauge velocity field can be defined for the Navier-Stokes equations when the fluid density is constant. These gauge fields are obtained from well defined boundary value problems and provide additional insight into the structure of the constant density NavierStokes equations. Of course, there is greater algebraic complexity in the gauge formulation. The decomposition has been illustrated with a discussion of the inactive motion outside a turbulent shear layer. 


\section{References}

[1] Seregin, G. Local regularity theory of the Navier Stokes equations. Handbook of mathematical fluid dynamics, IV, Friedlander, S. and Serre, D. (Eds)., pp 159-200, Elsevier, Amsterdam, 2007.

[2] Moulden, T. H. On properties of turbulence models. Advances in Fluid Mechanics VIII, Algarve, Portugal, pp 15-26, 2010.

[3] Chen, C-J and Jaw, S-Y. Fundamentals of turbulence modeling. Taylor and Francis, Washington, 1998.

[4] Wilcox, D. C. Turbulence modeling for CFD. 2nd Edition, DCW, LaCañada, 1998.

[5] Launder, B. E. and Sandham, N. D. (Eds) Closure Strategies for Turbulent and Transitional Flows. Cambridge University Press, Cambridge, 2002.

[6] Gatski, T. B. Constitutive equations for turbulent flows. Th. and Comp. Fluid Dyn. 18, pp 345-369, 2004.

[7] Gurtin, M. E. An introduction to continuum mechanics. Academic Press, New York, 1981.

[8] Frost, W. and Moulden, T. H. (Eds) Handbook of Turbulence. Plenum Press, New York, 1977.

[9] Moulden, T. H. A Uniqueness Theorem in Fluid Turbulence. Proc. 8th HEFAT Conference, Mauritius, 2011.

[10] Bradshaw, P. Irrotational fluctuations near a turbulent boundary layer. JFM, 27, pp 209-230, 1967.

[11] Speziale, C. G. Invariance of Turbulent Closure Models. Phys. Fluids, 22 pp 1033-1037, 1979.

[12] Wu, J-Z, Ma, H-Y and Zhou, M-D. Vorticity and Vortex Dynamics. Springer, Berlin, 2006.

[13] Weinan E and Liu, J-G. Finite difference schemes for incompressible flows in velocity-impulse density formulation. J. Comp. Phys. 130, pp 67-76, 1997.

[14] Wang, C. and Liu, J-G. Convergence of Gauge Method for Incompressible Flow. Math. Comp. 69, pp 1385-1407, 2000.

[15] Bradshaw, P. 'Inactive' motion and pressure fluctuations in turbulent boundary layers. JFM. 30, pp 241-258, 1967.

[16] Phillips, O. M. The irrotational motion outside a free turbulent boundary. Proc. Camb. Phil. Soc., 51, pp 220-229, 1955.

[17] Stakgold, I. Green's functions and boundary value problems. Wiley, New York, 1979.

[18] Goldstein, M. E. Aeroacoustics. McGraw Hill, New York, 1976.

[19] Schochet, S. The mathematical theory of the incompressible limit in fluid dynamics. Handbook of mathematical fluid dynamics, IV, Friedlander, S. and Serre, D. (Eds)., pp 159-200, Elsevier, Amsterdam, 2007.

[20] Moyal, J. E. The spectra of turbulence in a compressible fluid: eddy turbulence and random noise. Proc. Camb. Phil. Soc. 48, pp 329-344, 1952.

[21] Phillips, O. M. On the generation of sound by supersonic turbulent shear layers. JFM. 9, pp 1-28, 1960.

[22] Tam, C. K. W. On the noise of a nearly ideally expanded super sonic jet JFM. 51, pp 69-95, 1972. 